\title{
Amplitude Fluctuations in Small EPSPs Recorded from CA1 Pyramidal Cells in the Guinea Pig Hippocampal Slice
}

\author{
Rodney J. Sayer, ${ }^{1}$ Stephen J. Redman, ${ }^{1}$ and Per Andersen ${ }^{2}$ \\ 'Experimental Neurology Unit, The John Curtin School of Medical Research, The Australian National University, Canberra, \\ ACT 2601, Australia, and 'Institute of Neurophysiology, University of Oslo, Oslo 1, Norway
}

\begin{abstract}
EPSPs have been evoked in CA1 pyramidal cells by (1) activation of single CA3 neurons (unitary EPSPs), and (2) low-intensity stimuli to the CA1 stratum radiatum. Five unitary EPSPs were obtained; their mean peak amplitudes ranged from 85 to $275 \mu \mathrm{V}$ and 3 of the 5 showed fluctuations in amplitude that were too great to be attributed to baseline noise. After subtraction of the variance due to the noise, these EPSPs had coefficients of variation much higher than those reported for variability in the quantal EPSP in other preparations. These results suggest that intermittent transmitter release is a major cause of EPSP amplitude fluctuation at this synapse. A noise deconvolution technique based on a nonrestrictive model of transmitter release was applied to the EPSPs obtained in this study. For 2 of the EPSPs evoked by stratum radiatum stimulation, the amplitudes fluctuated between discrete values that were sufficiently separated with respect to the noise to be resolved by the deconvolution procedure. Quantal increments of 224 and $193 \mu \mathrm{V}$ were determined for the 2 EPSPs.
\end{abstract}

Pyramidal cells in the CA3 region of the hippocampus send at least one axonal branch (Schaffer collateral) into the stratum radiatum of area CA1 (Finch et al., 1983; Tamamaki et al., 1984), where they make excitatory synaptic connections with the apical dendrites of CA1 pyramidal cells. The in vitro slice technique has allowed substantial progress to be made on the pharmacology of synaptic transmission at this connection, in part motivated by interest in the phenomenon of long-term potentiation (LTP). However, little is known about some of the most basic structural and functional elements of the CA3-CA1 projection, especially the details of single cell-to-cell connections. In this study, we have made simultaneous intracellular recordings from CA3 and CA1 pyramidal cells to examine (1) the strength of input (in terms of somatic depolarization) of single CA3 pyramidal cells onto single CAl pyramidal neurons, (2) whether probabilistic release of transmitter occurs at the synapscs in this connection, and (3) whether fluctuations in amplitude of EPSPs can be explained by the statistical models

\footnotetext{
Received Apr. 25, 1988; revised July 28, 1988; accepted Aug. 5, 1988.

R.J.S. is the recipient of a National Health and Medical Research Council of Australia Postgraduate Research Scholarship. This work has been supported by the Norwegian Research Council for Science and the Humanities. We wish to thank Drs. F. Edwards, D. Daley, and D. Kullmann for their help with computing and statistics.

Correspondence should be addressed to Dr. S. J. Redman, Experimental Neurology Unit, The John Curtin School of Medical Research, The Australian National University, GPO Box 334, Canberra, ACT 2601, Australia.

Copyright (C) 1989 Society for Neuroscience $0270-6474 / 89 / 030840-11 \$ 02.00 / 0$
}

for quantal transmitter release proposed for other synapses (reviewed in Martin, 1966; McLachlan, 1978).

A nonrestrictive model of transmitter release has been assumed that allows either uniform or nonuniform probabilities of transmitter release at different release sites. Analysis of the fluctuations in the evoked EPSP does require several assumptions (also made in similar studies at other central synapses: Jack et al., 1981; Korn et al., 1982; Hess et al., 1987) viz., that variance in quantal size is negligible compared with the noise variance and that the processes generating the intracellular noise are statistically independent of the processes causing fluctuations in the evoked EPSP. The resolution of any procedure designed to determine quantal size and release probabilities is limitcd by the level of recording noisc and intraccllular noisc, which in CA1 pyramidal neurons is relatively high. In an attempt to obtain recordings with lower noise levels, a second series of experiments was carried out in which EPSPs were evoked in CA1 pyramidal neurons by low-intensity stimulation of the stratum radiatum using a protocol similar to that used by Hess et al. (1987). The stimulus intensity was adjusted to produce EPSPs similar in amplitude to those evoked by stimulating single CA3 neurons. Although this procedure carried the risk that more than one afferent might be activated and that if the stimulus intensity was close to threshold for these axons an additional cause of EPSP variability would be introduced, the method also had the advantage that a large number of CA1 neurons could be sampled to seek the lowest recording noise conditions.

These experiments have shown that EPSPs evoked in CA1 pyramidal neurons at the synapse(s) formed by a single $\mathrm{C} \Lambda 3$ neuron range in amplitude from 85 to $275 \mu \mathrm{V}$, and the results suggest that some of these EPSPs fluctuate in amplitude due to intermittent transmitter release, that the quantal amplitude has an upper limit of about $200 \mu \mathrm{V}$ and is probably much smaller than this for most CA3-CA1 synapses.

\section{Materials and Methods}

Brains were removed from 600-900 gm guinea pigs deeply anesthetized with ether and were chilled in artificial cerebrospinal fluid (ACSF) at $2-4^{\circ} \mathrm{C}$. The hippocampus was dissected out from one side, and $450 \mu \mathrm{m}$ slices were cut with a tissue chopper. The hippocampus was aligned on the chopper so that the slices were cut parallel to the lamellae, $20^{\circ}-30^{\circ}$ from the transverse plane with the rostral end of the blade turned medially. The slices were transferred onto a net covered with lens tissue in a recording chamber where they were maintained at $32-33^{\circ} \mathrm{C}$ between ACSF and humidified gas $\left(95 \% \mathrm{O}_{2} / 5 \% \mathrm{CO}_{2}\right)$. The ACSF consisted of (in mM): $\mathrm{NaCl}, 124 ; \mathrm{KCl}, 2 ; \mathrm{MgSO}_{4}, 2 ; \mathrm{CaCl}_{2}, 2 ; \mathrm{KH}_{2} \mathrm{PO}_{4}, 1.25 ; \mathrm{NaHCO}_{3}$, 26; and glucose, 11 , saturated with $95 \% \mathrm{O}_{2} / 5 \% \mathrm{CO}_{2}$.

Simultaneous CA3-CA1 intracellular recordings. Recording electrodes were pulled from 1.5-mm thick-walled glass (GC150OF-15, Clark 

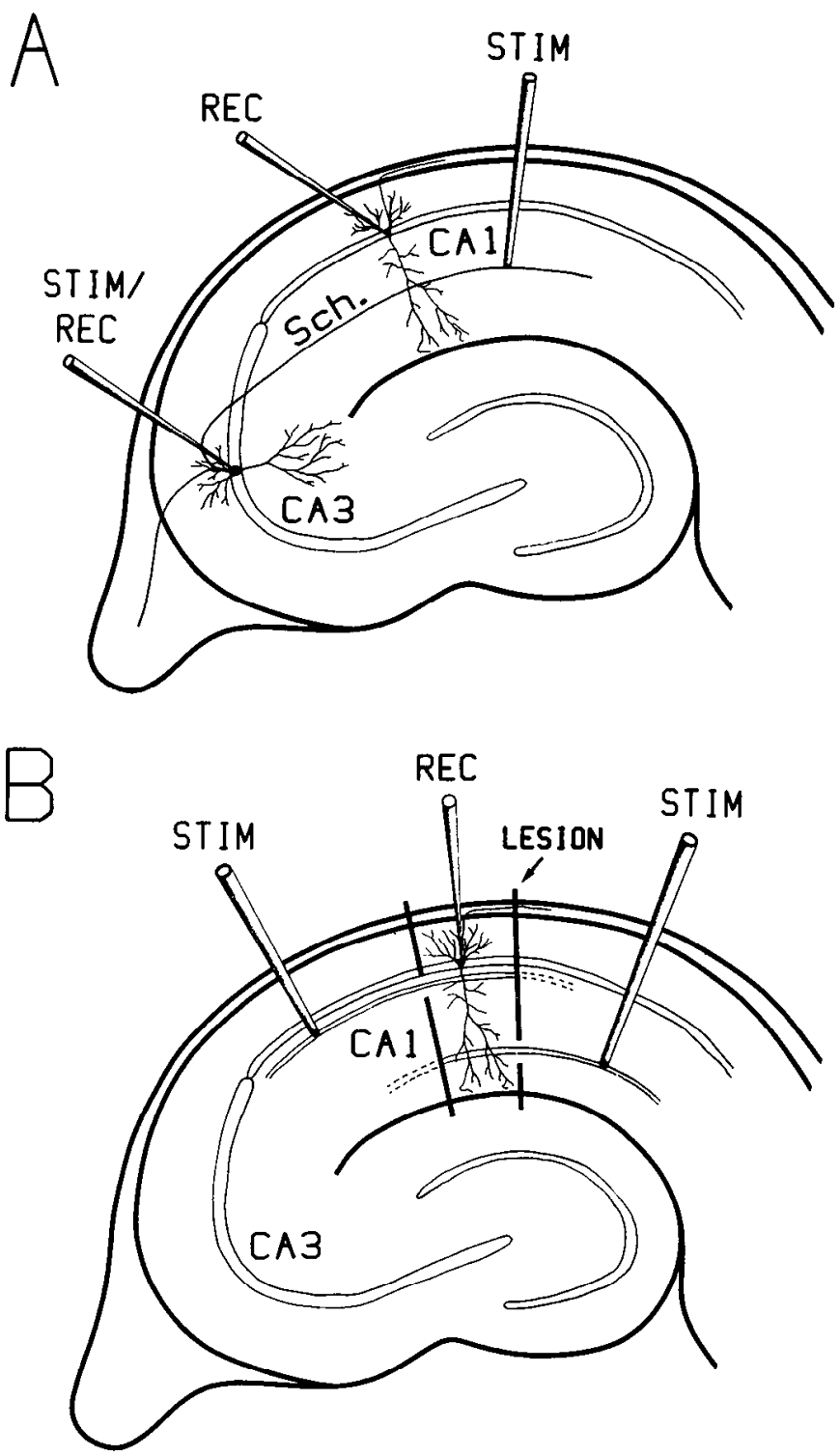

Figure 1. Experimental protocols. $A$, For the simultaneous CA3/CA1 recordings, a pyramidal cell was impaled in the CA3 stratum pyramidale. The presence of a Schaffer collateral $(S c h$.) in the CA1 stratum radiatum could be checked by antidromically activating the collateral with a stimulating electrode (STIM) and this fiber could then be orthodromically discharged by injecting a current pulse into the CA3 neuron through the intracellular recording electrode (STIM/REC). CA1 pyramidal cells were impaled by an electrode in the CA1 stratum pyramidale. $B$, For the fluctuation analysis experiments, pyramidal cells were impaled by a recording electrode $(R E C)$ in the CA1 stratum radiatum. EPSPs were evoked by stimulating electrodes (STIM) positioned for alternately exciting fibers running distally and proximally (with respect to the stratum pyramidale) in the stratum radiatum. Synaptic input to the cells was restricted to the proximal and distal regions by cuts made across the slice (solid lines), leaving bridges of intact tissue $50 \mu \mathrm{m}$ wide.

Flectromedical Instruments), were shielded with aluminum foil, and had resistance of 80-120 M $\Omega$ when filled with $2 \mathrm{M}$ potassium methylsulfate. To improve the chances of finding a connecting CA3-CA1 pair, we first looked for a $\mathrm{CA} 3$ cell that could be antidromically activated by a stimulating electrode placed in the $\mathrm{CAl}$ stratum radiatum (Fig. $\mathrm{I} A$ ). This ensured that the CA3 neuron had a collateral running in the plane of the slice through the CA1 apical dendritic field. If a CA1 cell had already been impaled, CA3 cells were usually tested even if they could not be antidromically activated. Pyramidal cells were impaled in the
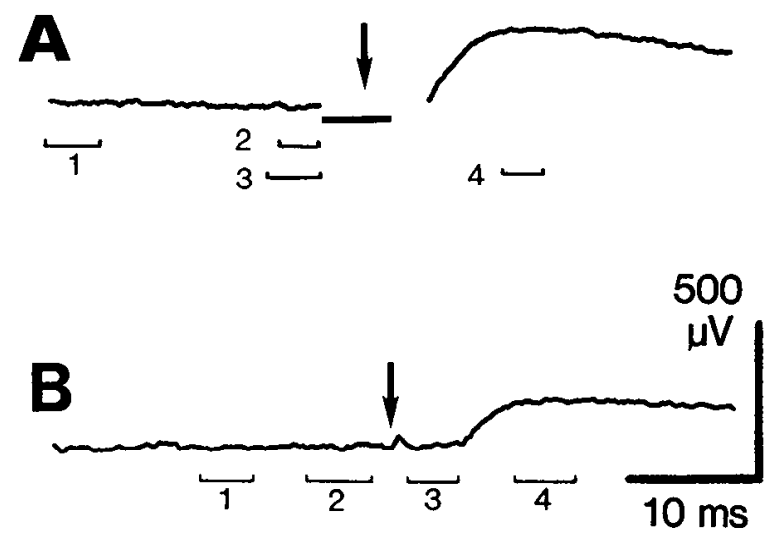

Figure 2. A, Average of 980 records from the CA1 cell of CA3-CA1 pair 3 (Table 1), showing the regions selected for noise and noise-contaminated EPSP amplitude measurement (numbered 1-4). The arrow indicates the time of the peak of the action potential in the CA3 cell. $B$, Average of 1718 records evoked by proximal stimulation of cell 1 (Table 2). The regions selected for measurement of noise and noisecontaminated EPSP amplitudes are numbered $1-4$. The arrow indicates the time of the stratum radiatum stimulus.

CA1 stratum pyramidale $200-1000 \mu \mathrm{m}$ from the CA3/CA1 border. Once stable intracellular penetrations were achieved (for the CAl cell this required the membrane potential to be more negative than $55 \mathrm{mV}$, spike $>70 \mathrm{mV}$, input resistance $>20 \mathrm{M} \Omega$ ), the CA3 neuron was stimulated at $2 \mathrm{~Hz}$ by an intracellular depolarizing current pulse. The depolarizing pulse was adjusted $(0.5-2.5 \mathrm{nA}, 3-5 \mathrm{msec})$ to evoke a single action potential. Sometimes a small steady hyperpolarizing current $(0.1-$ $0.5 \mathrm{nA}$ ) was applied to the CA3 cell to prevent spontaneous firing. If on-line averaging showed any postsynaptic response, as many records (usually $60 \mathrm{msec}$ duration) as possible were digitized at $4 \mathrm{kHz}$ and saved on disk while the membrane potentials in each cell remained satisfactory. After withdrawing the electrode from the CAl cell, an extracellular average was taken while stimulating the $\mathrm{CA} 3$ neuron to check for any extracellular field and for subtraction of the electrode coupling artifact. Small lesions were made at the recording sites, and after overnight fixation in $3 \%$ glutaraldehyde the slices were stained with thionine for microscopic localization of the recording sites with respect to the CA3/ CAl border.

Presynaptic records were checked to ensure that CA3 cells discharged only once for each current pulse. If there was multiple firing or a failure to discharge, the corresponding CAI record was not included in the analysis. Scaled extracellular averages were subtracted from each record to minimize the duration of the coupling artifact.

In each experiment we attempted to record 1000 or more individual responses. These responses were averaged, using the time of the peak of the CA 3 action potential as $t=0$ for each record to minimize latency variations. The averaged EPSP in Figure $2 A$ was obtained in this way, and 2 regions were selected for the measurement of peak amplitude (regions 3 and 4 ). The baseline region (3) immediately preceded the coupling artifact (blanked out in Fig. $2 A$ ) and a peak region (4) straddled the maximum of the EPSP. Region 4 was chosen such that throughout this period the amplitude of the averaged EPSP always exceeded $90 \%$ of its peak value. These regions were usually of $3-4 \mathrm{msec}$ duration. For each response, the average voltages over regions 3 and $4\left(V_{3}\right.$ and $\left.V_{4}\right)$ were calculated, and $V_{4}-V_{3}$ was used to construct an amplitude histogram of the noise-contaminated EPSP (Fig. 4). Similarly, a histogram of the noise voltage present during the recording was obtained by selecting regions before the coupling potential (regions 1 and 2 in Fig. $2 A$ ) to have identical durations to regions 3 and 4 , respectively, and to be separated by the same period as regions 3 and 4 . The average voltages in regions 1 and $2\left(V_{1}\right.$ and $\left.V_{2}\right)$ were calculated for each response, and $\mathrm{V},-\mathrm{V}$, was used to construct the noise amplitude histogram. The histograms of the noise voltage and the noise-contaminated EPSP were used to calculate the noise-free EPSP probability density using a deconvolution technique described below.

The time course of the SD of each EPSP was calculated by subtracting the mean EPSP from each evoked response, squaring the difference, subtracting the noise variance record, and then taking the square root 
of the result (Jack et al., 1981). The SD time courses are shown in Figure 3.

EPSPs evoked in CA1 neurons by stimulation of stratum radiatum. In these experiments, cuts were made to restrict the Schaffer collateral/ commissural input to regions of the stratum radiatum which were either proximal or distal with respect to the stratum pyramidale (Fig. $1 B$ ). The cuts were made using the method of Andersen et al. (1980), and the proximal and distal bridges of intact tissue were $<50$ and $350-450 \mu \mathrm{m}$ from the stratum pyramidale, respectively. Similar protocols for proximal and distal microstimulation, but without making cuts, are described by Andersen et al. (1987) and Turner (1988). One apparent advantage of the microsurgery was that obvious contamination of the EPSPs by IPSPs was only rarely seen, perhaps because the inhibitory interneuron collaterals in the strata pyramidale and oriens are cut. In nonlesioncd slices, IPSPs can be evoked at very low stimulus intensities (unpublished observations, and Turner, 1988). However, the possibility of IPSP contamination could not be completely ruled out in this study. Bicuculline methiodide $\left(10^{-6} \mathrm{M}\right.$, Sigma) was added to the ACSF in some experiments (cell 1 and cell 3 in Table 2).

Monopolar stimulating electrodes were pulled from capillary tubing, and the tips were broken back and heat-polished to $5-15 \mu \mathrm{m}$ before being filled with $1 \mathrm{M} \mathrm{NaCl}$ in gelatin. The shanks were coated with Sylgard (Dow Corning) and the shafts shielded with conductive paint. When positioned on the surface of the slice, these electrodes produced a minimum of trauma, and by reducing fluid accumulation around the tips, they ensured that stimulus current densities remained constant over an extended period. Recording electrodes were made from 1.5$\mathrm{mm}$ thin-walled glass (GC150TF-15, Clark Electromedical Instruments), filled with $2 \mathrm{M}$ potassium methylsulfatc and bevclcd to a resistance of 20-40 M $\Omega$. These relatively low resistance electrodes gave lower levels of recording noise but did reduce the yield of stable penetrations and in general resulted in membrane and action potentials of slightly lower amplitude than were obtained with finer electrodes.

Once stable intracellular penetrations were achieved (membrane potential more negative than $-55 \mathrm{mV}$, spike $>60 \mathrm{mV}$, input resistance $>15 \mathrm{M} \Omega$ ), EPSPs were evoked by alternately activating fibers passing through each bridge. Stimuli were negative constant-current pulses 100 $\mu \mathrm{sec}$ in duration delivered at either 1 or $2 \mathrm{~Hz}$ (per input) through electrodes $800-1300 \mu \mathrm{m}$ from the recording site. The stimulus currents (5$30 \mu \mathrm{A}$ ) were adjusted to evoke the smallest EPSP that could be maintained at a stable amplitude when averaged over epochs of $30 \mathrm{sec}-1$ min. Usually, the stimulus intensity was increased to slightly greater than that which produced the smallest EPSP discriminable by avcraging. We presume that the smallest detectable EPSPs are less stable because they are evoked at the stimulus threshold for axon recruitment and are therefore sensitive to the slightest changes in stimulus current density. For each cell we attempted to record 2000 or more individual responses for each input (proximal and distal). Successive EPSPs, and a prestimulus region for measuring the probability density distribution of the noise, were digitized at $4 \mathrm{kHz}$ and saved on disk. After withdrawal from the cell an extracellular average was recorded for subtraction of any stimulus-evoked field potential.

Sequential sets of 100 records were averaged and plotted against time to check that no drift had occurred in the EPSP amplitude during recording. As for the CA3-CA1 EPSPs, all responses were averaged, and the average was used to select time intervals for measurement of peak EPSP and noise voltage (Fig. $2 B$ ). The same procedure as that used for calculating the peak amplitude of the CA3-CA1 EPSP was followed, except the absence of the coupling artifact meant that the baseline for the EPSP could be measured in a period immediately preceding its rising phase. This is an important advantage, as the noise variance is reduced by decreasing the interval between regions 1 and 2 . Histograms of the evoked EPSP and the noise were constructed and then used in a deconvolution calculation. Although EPSPs evoked by proximal and distal stimulation were analyzed separately, both their preartifact regions were used for noise measurements as this allowed the noise voltage histograms to be constructed from twice as many records as the corresponding EPSP histograms. However, the noise histograms usually differed for proximal and distal EPSPs recorded in the same cell because the periods 3 and 4 were separated by different durations and extended over different periods.

Deconvolution procedures. The probability density of EPSP amplitudes was obtained by deconvolving the noise-contaminated EPSP amplitude histogram with the histogram for the noise voltage using a recently developed deconvolution procedure. The statistical task was to obtain the maximum likelihood estimates of the mixing proportions and voltages of the different discrete amplitudes making up the fluctuation of the EPSP amplitude. The maximum likelihood estimator (MLE), a variant of the Estimation-Maximization (E-M) algorithm (Dempster et al., 1977) first described by Hasselblad (1966), was applied in 2 stages. Briefly, the noise histogram was approximated by a continuous monotonic distribution made up from the sum of 2 Gaussian curves, using the MLE to find their optimal probabilities, SDs, and positions relative to zero on the voltage axis. (A slight positive skewness in some of the noise histograms made a 2-Gaussian fit superior to a single-Gaussian representation.) The mixture problem was then solved by applying the MLE to the histogram of the evoked EPSP, this time treating the noise fit as the contaminating distribution and allowing only the probabilities and positions of the underlying discrete amplitudes of the EPSP to vary. In all other respects, the technique follows the procedures outlined in Jack et al. (1981) and Wong and Redman (1980). The discrete amplitudes determined by this procedure are not constrained to be equally spaced, nor are the probabilities assigned to them forced to conform to any overall probability distribution (e.g., the binomial distribution), except that the sum of probabilities for all values must be equal to one. All discrete amplitudes were included in the result, regardless of their probabilities of occurrence. However, when calculating the mean separation between adjacent discrete amplitudes (mean quantal amplitude), only those amplitudes with probabilities of occurrence $>0.05$ were included, as amplitudes with smaller probabilities are likely to be unreliably located.

\section{Results}

\section{Simultaneous CA3-CA1 recordings}

One hundred and ninety CA3-CA 1 pairs were tested in 36 slices (16 experiments). For 5 of these pairs, EPSPs became apparent after averaging about 100 responses in the CAl neuron. The number of records obtained for each pair is indicated in Table 1. The averages of these EPSPs are shown in Figure 3 (heavy lines), and their average peak amplitudes ranged from 85 to 275 $\mu \mathrm{V}$ (Table 1). The CA1 neuron membrane potentials were between -59 and $-64 \mathrm{mV}$ during these recordings. The locations of the pre- and postsynaptic cells, in CA3 and CA1, respectively, were confirmed from thionine-stained sections and are illustrated in Figure 3. In 3 of the 5 pairs, the CA3 neurons were antidromically activated by stimuli $(25-70 \mu \mathrm{A})$ in the midradiatum of $\mathrm{CA} 1$; in one case, it was assumed that no antidromic response was obtained because a faulty stimulating electrode produced inadequate current (pair 1); and for pair 3, the CA3 cell could be discharged antidromically only after the stimulating electrode was relocated to $50 \mu \mathrm{m}$ on the CA3 side of the CA1 recording electrode. For antidromic stimulation of this CA3 cell, high stimulus currents were needed $(450 \mu \mathrm{A})$, and the threshold was similar over proximal radiatum, stratum pyramidale, and stratum oriens. This suggests either that the afferent fiber ran deep in the slice and could have made contact with either the apical or basal dendrites of the CA1 neuron or that there were several axonal branches at different levels.

After subtraction of the CA1 extracellular average, some electrode coupling artifact usually remained to obscure the rising phase of the EPSPs. The time courses for these EPSPs were therefore not examined. However, the EPSP peaks were always beyond the tail of the coupling artifact, so the peak amplitude distributions and mean peak amplitudes were unaffected.

The conduction times of the antidromic spikes from CAl radiatum to CA3 were generally $1-2 \mathrm{msec}$, so it was not surprising that the onsets of the CAl EPSPs were usually within the coupling artifact. Note that one EPSP (no. 4) had a latency a few milliseconds longer than the others. This delay is probably due to a more slowly conducting fiber. Variation of conduction velocities would be consistent with the 3-fold range of fiber 

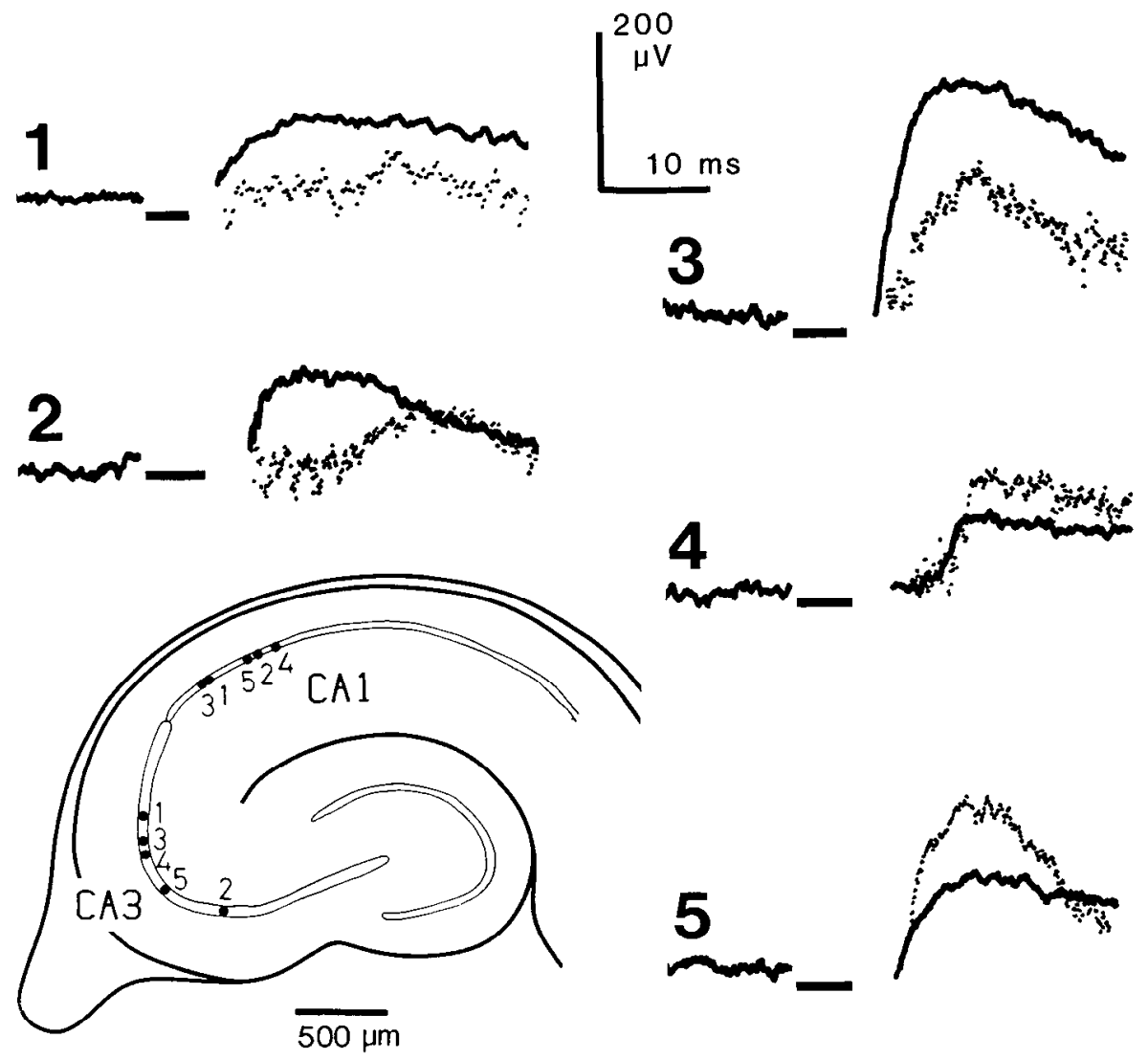

diameters $(0.08-0.30 \mu \mathrm{m})$ reported for rat stratum radiatum (Westrum and Blackstad, 1962).

Figure 4 shows the histograms of the evoked EPSP peak amplitude and the noise voltage for 2 CA3-CA1 EPSPs (nos. 2 and 3, Table 1). For all 5 EPSPs the noise level on the CA1 records was too large to allow reliable resolution of discrete EPSP amplitudes. The high noise levels were caused by using fine electrodes to obtain a high yield of stable intracellular penetrations and also by the need to shift the baseline region away from the initial rising phase of the EPSP, thereby avoiding the CA3 spike coupling artifact. However, some useful information can be deduced from these results. The histograms in Figure $4 A$ and $4 B$ differ in that the noise would seem to account for all of the variability in the EPSP histogram in Figure $4 A$, while it clearly cannot account for all of the variability in the EPSP histogram in Figure $4 B$. The SDs for the noise $\left(\sigma_{\mathrm{N}}\right)$ and for the EPSP $\left(\sigma_{\mathrm{E}}\right)$

\begin{tabular}{llllll}
$\begin{array}{l}\text { Table 1. } \\
\text { pairs }\end{array}$ & Characteristics of CA1 recordings for contacting CA3/CA1 \\
Cell & $\begin{array}{l}\text { Number of } \\
\text { records }\end{array}$ & $\begin{array}{l}\bar{\nu} \\
(\mu \mathrm{V})\end{array}$ & $\begin{array}{l}\sigma_{\mathrm{N}} \\
(\mu \mathrm{V})\end{array}$ & $\begin{array}{l}\sigma_{\mathrm{E}} \\
(\mu \mathrm{V})\end{array}$ & $\mathrm{CV}$ \\
\hline no. & 1280 & 96 & 459 & 435 & - \\
\hline 1 & 847 & 113 & 198 & 219 & - \\
2 & 980 & 275 & 219 & 289 & 0.68 \\
3 & 800 & 85 & 177 & 232 & 1.76 \\
4 & 1127 & 90 & 325 & 392 & 2.44 \\
5 & &
\end{tabular}

$\overline{\bar{v}}$, mean amplitude of EPSP; $\sigma_{N}$, standard deviation of noise histogram; $\sigma_{\digamma}$, standard deviation of noise-contaminated EPSP histogram; $\mathrm{CV}$, coefficient of variation $\left[\left(\sigma_{\mathrm{F}}^{2}-\sigma_{\mathrm{N}}^{2}\right)^{1 / 2 / \overrightarrow{\mathrm{v}}}\right]$. pair 3 (Table 1 ).
Figure 3. Averaged single-fiber EPSPs from CAl pyramidal cells (solid traces). Capacitive coupling artifacts from the CA3 electrode have been blanked out, and the bars indicate the duration of current injection in the $\mathrm{CA} 3$ cell. The number of records used to construct each average is given in Table 1 . The dotted traces indicate the time course of the SD for each EPSP. Variance records have been calculated for both preand poststimulus regions of the traces, and these SD records are the square roots of the differences between them. The locations of the CA3 and CA1 neurons, with respect to the CA3/CA1 border, are shown for each of the contacting pairs in the sketched hippocampal slice.

are listed in Table 1 for all 5 EPSPs, and the time courses of the SD records for these EPSPs are shown in Figure 3. These records for EPSPs 1 and 2 showed no obvious deviation from baseline throughout the early time course of these EPSPs (including the peak region).

A superficial interpretation of these records would suggest that after the variability caused by additive noise is removed, EPSPs 1 and 2 do not fluctuate in amplitude, implying no quantal variability and no fluctuations in the release of transmitter from trial to trial. The histograms of the noise and EPSP in Figure $4 A$ can be tested against the null hypothesis that they have been sampled from distributions having the same SD. This hypoth-

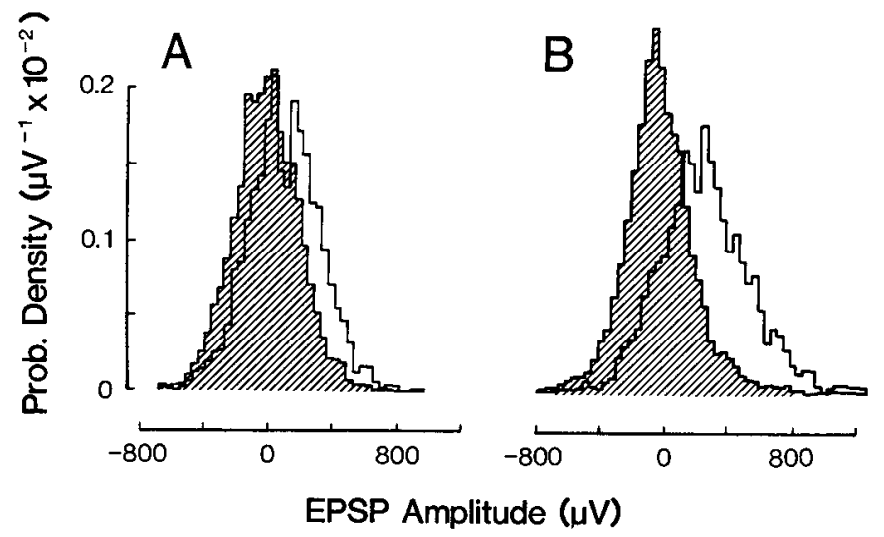

Figure 4. Noise (shaded) and noise-contaminated EPSP amplitude histograms $(A)$ for CA3-CA1 pair 2 (Table 1) and $(B)$ for CA1-CA3 
Figure 5. Examples of single records and the averages of all records for the proximally and distally stimulated EPSPs in cell 1 (Table 2). Averaged extracellular records have been subtracted. Arrows indicate the time of stimulus.

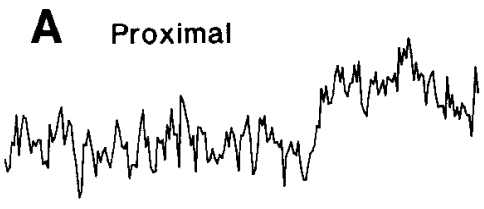

B Distal
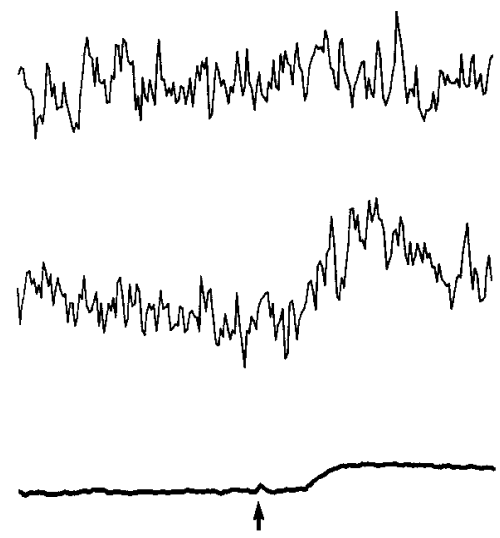

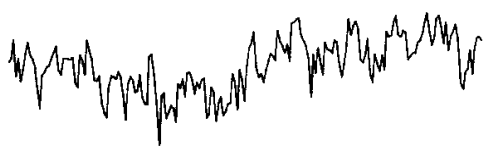

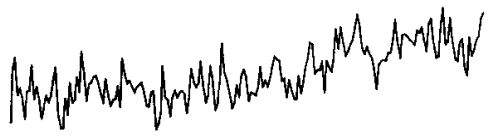

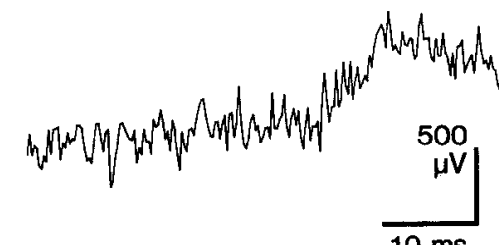

$10 \mathrm{~ms}$

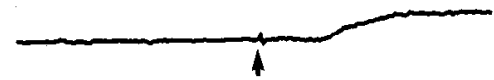

esis can be tested using Fisher's $z$-approximation to the $F$-distribution (Johnson and Kotz, 1970, p. 81), where $z=\sqrt{N} \log$ $\left(\sigma_{\mathrm{N}} / \sigma_{\mathrm{L}}\right)$ and $N$ is the sample size. We find $z=-1.27$ for the EPSP in Figure $4 A$ (EPSP 2 in Table 1), which is not significant at the 0.05 confidence level; this indicates that we cannot reject the null hypothesis. But this would also happen if $\sigma_{\mathrm{E}}$ was as large as $232 \mu \mathrm{V}$, and it could be that $\sigma_{\mathrm{E}}$ has 2 components. One would be the recording and intracellular noise $\left(\sigma_{\mathrm{N}}=198 \mu \mathrm{V}\right)$ and the other would be intrinsic variability in the EPSP $\left[\left(\sigma_{\mathrm{E}}^{2}-\sigma_{\mathrm{N}}^{2}\right)^{1 / 2}=\right.$ $121 \mu \mathrm{V}]$. That is, while we cannot reject the null hypothesis that both the noise and the recorded EPSP have been sampled from distributions with the same variance, the high noise level and finite sampling still allow the possibility that some of the EPSP variability arises from quantal variability and/or fluctuations in the number of quanta released.

Another approach to this problem is to examine the time coursc of the SD record during the EPSP (no. 2 in Fig. 3). The peak-to-peak variation in this record corresponds to a peak-topeak variation in the difference between $\sigma_{\mathrm{N}}^{2}$ and $\sigma_{\mathrm{E}}^{2}$ of about $(125 \mu \mathrm{V})^{2}$; i.e., there could be an intrinsic SD associated with the EPSP with a peak value of about $150 \mu \mathrm{V}$, but this would not be resolvable by eye from the SD record.

The other EPSP (no. 1) in Figure 3, which appears to have no variability other than that due to noise, also cannot be rejected at the 0.05 confidence level using the same null hypothesis that the noise and the EPSP have been sampled from distributions with the same variance, but the corresponding margin for intrinsic EPSP variation is $249 \mu \mathrm{V}$, or $150 \mu \mathrm{V}$ when calculated from the SD record. However, the $z$-values for EPSPs 3, 4 , and 5 in Figure 3 were significant at the $0.001,0.001$, and 0.01 levels of confidence, respectively, and for these EPSPs the null hypothesis can be rejected.

These results are important because they demonstrate that some CA3-CA1 EPSPs have intrinsic amplitude variations from trial to trial when the CA3 neuron is reliably discharged on each trial. They also suggest that this variability is at least partially due to variations in the number of quanta released. The reason for this last statement is that the coefticients of variation (CV) for EPSPs 3, 4, and 5 are $0.68,1.76$, and 2.44, respectively
(Table 1). The smallest CV possible for a quantal EPSP would then be 0.68 , and this would require EPSP no. 3 to be generated by the invariant release of a single quantum. (It would be $0.68 / \sqrt{\mathrm{N}}$ for the invariant release of $\mathrm{N}$ quanta, while the scaling is more complex when variable numbers of quanta can be released on each trial.) As the largest reported quantal CV is 0.3 , measured at the neuromuscular junction (Martin, 1977), it seems unlikely that all the variability associated with the three EPSPs can be accounted for by variation in quantal size, and the number of quanta released is likely to vary on each trial.

\section{Fluctuation analysis of EPSPs evoked by stimulation of stratum radiatum}

It was apparent from the preceding results that unless the recording noise level was reduced considerably, it would be impossible to resolve fluctuations in EPSP amplitude. The noise level could be reduced by lowering the electrode resistance and by sampling many different CAl neurons to find those with the least spontaneous synaptic activity. This approach could not be used for the CA3-CA1 EPSPs because of the low probability of finding a connection. We turned to microstimulation of the stratum radiatum, combined with microsurgery, which left thin strands of tissue intact (Fig. $1 B$ ). However, the EPSPs evoked by maximal stimulation on the distal sides of these tissuc bridges had large peak amplitudes (1-10 mV), suggesting that many axons in the bridge remained functional. The discrete amplitudes of the large EPSP evoked by stimulating the remaining axons would be unresolvable, so we resorted to submaximal stimulation as the most reliable and stable method for obtaining large samples of EPSPs with average peak amplitudes of about the same magnitude as unitary EPSPs. These EPSPs were then analyzed using the deconvolution procedure in an attempt to determine the size of quantal EPSPs. A similar experimental approach was used by Hess et al. (1987).

Records were accepted for fluctuation analysis from 5 out of 35 CA1 pyramidal cells, on the basis of low recording noise (SD of noise amplitude distribution $<140 \mu \mathrm{V}$ ), stationarity of EPSP amplitude throughout the recording period and a sufficient number of records (range, 1718-4817). Proximally and distally evoked 


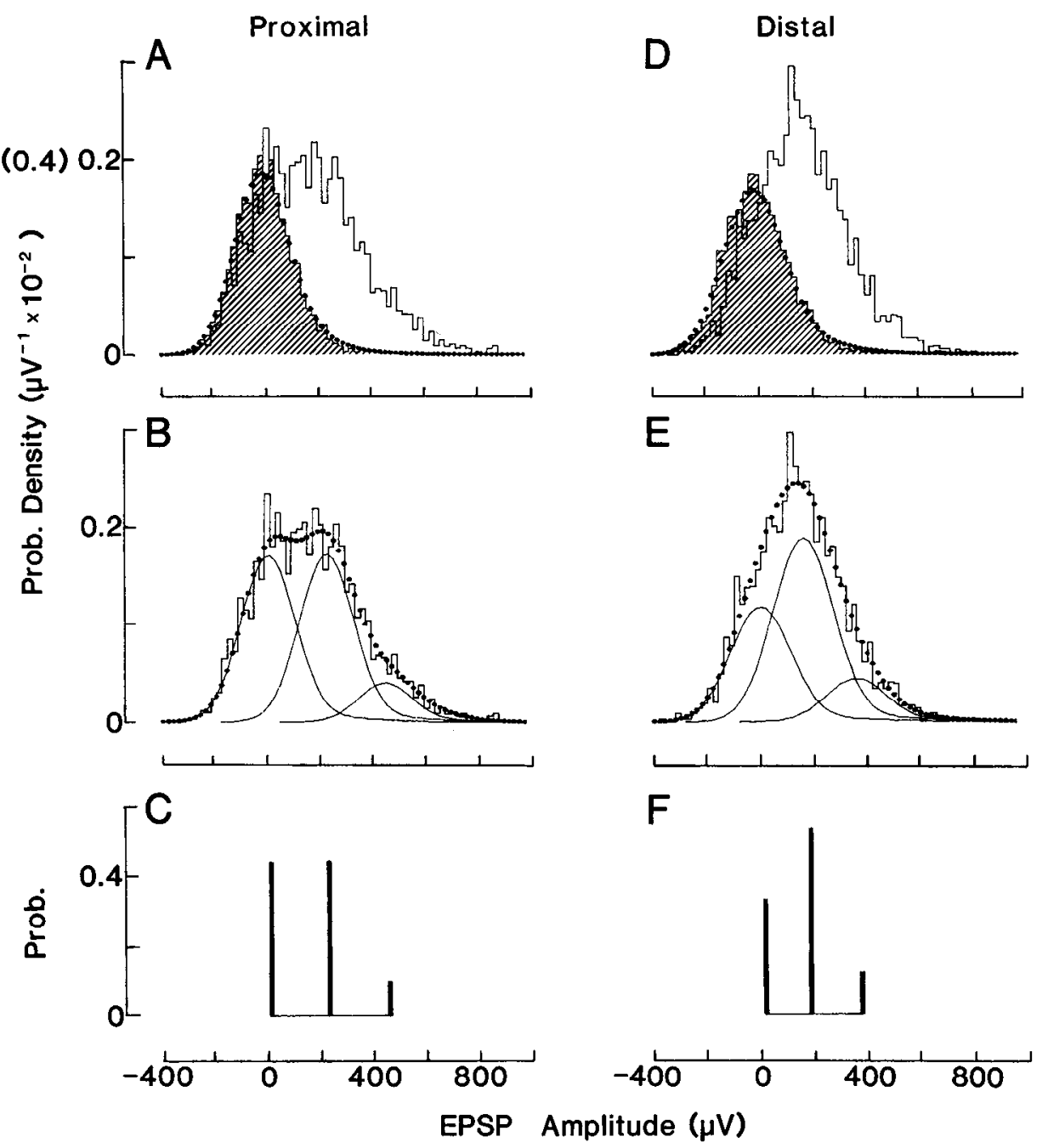

Figure 6. Deconvolution analysis for cell 1 (Table 2). $A$, Noise amplitude histogram (shaded) superimposed on the noise-contaminated EPSP amplitude histogram, for the proximally stimulated EPSP. The filled circles indicate a normal distribution fitted to the noise amplitude histogram, used as the noise distribution by the deconvolution procedure. Note the smaller vertical scale for the noise histograms $(0.4)$. Bin width is $20 \mu \mathrm{V}$ for both histograms. $B$, Filled circles indicate the reconvolved deconvolution result for the proximally clicitcd EPSP and wcre obtained by summing the 3 underlying Gaussian curves. These points have been superimposed upon the noisecontaminated EPSP amplitude histogram. The 3 Gaussian distributions have the same $\operatorname{SD}\left(\sigma_{\mathrm{N}}\right)$ as the measured noise distribution, and the mean values are equal to each of the discrete amplitudes and the areas are equal to the probabilities associated with the discrete amplitudes. $C$, Deconvolution results for this EPSP, shown as a sequence of discrete amplitudes and associated probabilities. $D-F$, These correspond to $A-C$ but are for the distally stimulated EPSP in the same cell.
EPSPs were recorded in each cell. Mean EPSP peak amplitudes ranged from 95 to $395 \mu \mathrm{V}$.

Figure 5 shows examples of single records, and the averages of all records, for the proximally and distally stimulated EPSPS in one cell. In agreement with previous results on small synaptic responses in CA1 (Turner, 1988), the averaged EPSP evoked by the proximal input had the shorter $10-90 \%$ rise time $(5.4$ msec vs. $7.3 \mathrm{msec}$ for the distally evoked EPSP). The histograms of the noise voltage and of the noise-contaminated EPSP obtained by averaging each record over the peak and baseline regions are shown for this cell in Figure $6, A, D$. Deconvolution analysis indicated that the proximally stimulated EPSP had discrete amplitudes $(\mu \mathrm{V})$ associated probabilities of $16 / 0.44$, $240 / 0.44$ and $463 / 0.12$, as shown in Figure $6 C$. The contribution that each of these discrete amplitudes made to the measured histogram is shown in Figure $6 B$. Gaussian noise distributions, with SDs equal to that of the noise, and with means equal to the different discrete amplitudes, are scaled to have an area equal to the probability associated with each discrete amplitude. The sum of these distributions (filled circles) is the best fit to the measured histogram, using the maximum likelihood criterion. Individual EPSPs evoked in this neuron by distal stimulation, as well as the average of all responses, are illustrated in Figure $5 B$, while the deconvolution results for this EPSP are shown in Figure $6, E, F$. The discrete amplitudes $(\mu \mathrm{V}) /$ probabilities given by the deconvolution procedure were $28 / 0.34,196 / 0.54$, and $386 / 0.13$.

The interpretation of these results depends upon how accurately the deconvolution method can resolve the fluctuations between discrete amplitudes under a variety of noise and sample size conditions. Figure 7 shows the results of a Monte Carlo simulation of the deconvolution procedure where the noise-free EPSP distribution (indicated by vertical bars) consisted of 3 discrete amplitudes with probabilities $0.4,0.4$, and 0.2 (for increasing amplitudes). Samples drawn from a distribution of intracellular noise were added to samples drawn from the simulated EPSP distribution. The SD of the noise $\left(\sigma_{\mathrm{N}}\right)$ was kept constant, while the quantal increment $\left(\bar{v}_{1}\right)$ was altered in different simulations (Fig. $7, A-D$ ) to represent different signal-to-noise conditions $\left(\bar{v}_{1} / \sigma_{\mathrm{N}}\right)$. Thirty deconvolutions were performed for each set of conditions, and the peak amplitudes/probabilities are indicated by filled circles. When the sample size is 2000 and $\bar{v}_{1} / \sigma_{\mathrm{N}}$ is 2.0 (Fig. $7 \mathrm{~A}$ ), the original EPSP distribution is recovered reliably in almost all deconvolutions, as seen by the clustering of the filled circles around the tops of the vertical lines. This situation quickly deteriorates if the sample size is reduced (Fig. $7 B$ ) or if $\bar{v}_{1} / \sigma_{N}$ decreases (Fig. 7, C, D). From these simulations (and others of a similar kind; Jack et al., 1981; Clements et al., 1987) we have determined that if the sample size is 2000 records, 3 discrete amplitude values separated by more than $1.6 \sigma_{\mathrm{N}}$ will 
Figure 7. Testing the deconvolution procedure by a Montc Carlo simulation. The true noise-free EPSP amplitude distributions are indicated by the vertical bars. The filled circles represent the deconvolution results (discrete amplitude values and probabilities). Thirty simulations were deconvolved for each of 4 sample size $(N)$ and signal/ noise $\left(v_{1} / \sigma_{\mathrm{N}}\right)$ conditions. In $A, C$, and $D$, the separation of the discrete amplitudes was decreased from 2.0 to 1.6 and $1.3 \sigma_{\mathrm{N}}$, respectively, for a constant sample size of 2000 EPSPs. $B$, The separation of discrete amplitude values is the same for $A$, but the sample size has been decreased to 500 . The noise samples were obtained from intracellular records.
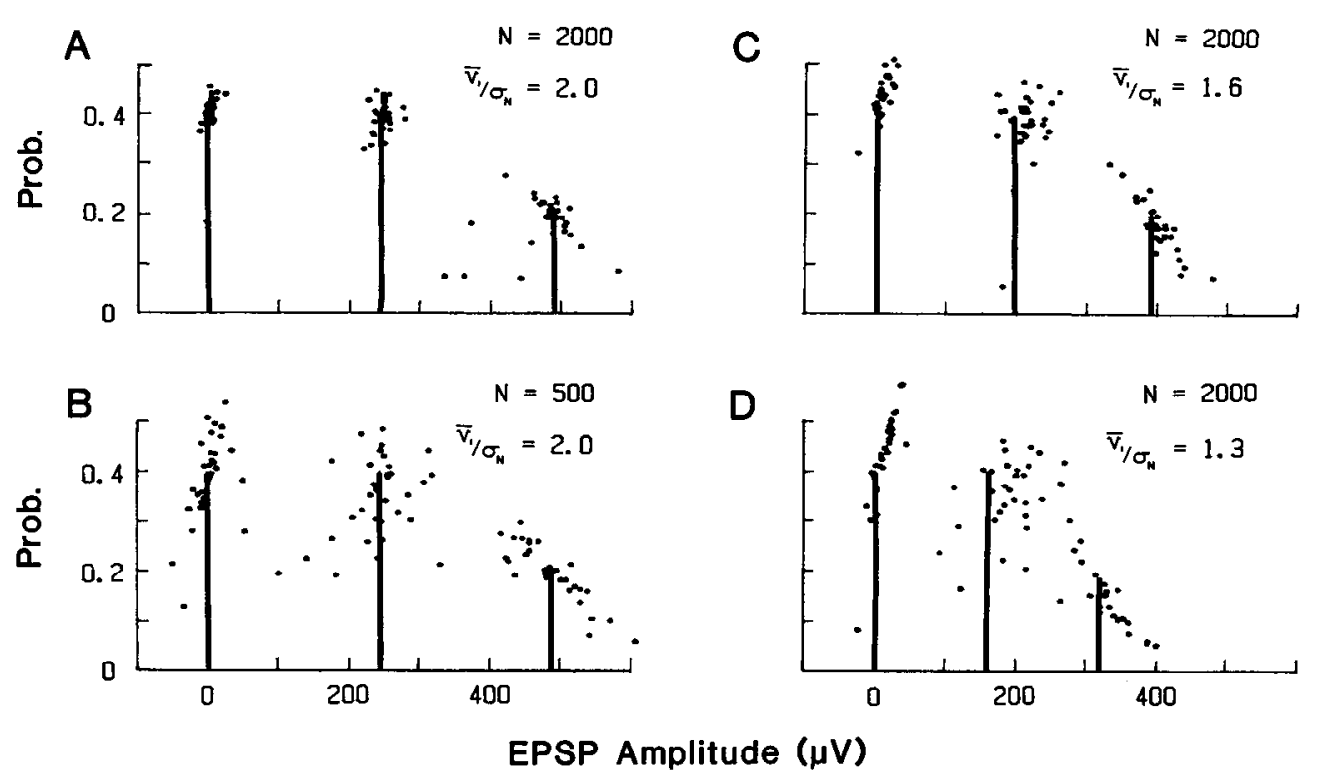

be located with sufficient accuracy (where the mean increment between adjacent peak amplitude is determined to within $\pm 10 \%$ of its correct value on more than $80 \%$ of the trials). On the occasions when the result does err by more than $10 \%$ from the truc mcan, the crror is almost always a "safe" onc, bccausc it results from the introduction of extra discrete amplitudes between the true values. These reduce the mean separation of discrete amplitudes to below $1.6 \sigma_{\mathrm{N}}$, and the result is rejected as unreliable (see below). When the true discrete amplitudes are separated by less than $1.6 \sigma_{\mathrm{N}}$, the deconvolution algorithm can fail to resolve these amplitudes reliably. It sometimes combines adjacent discrete amplitudes to give false amplitude values separated by up to $1.6 \sigma_{\mathrm{N}}$. Thus, when the separation between adjacent peak amplitudes is unknown, answers with amplitudes separated by less than $1.6 \sigma_{\mathrm{N}}$ should be considered suspect and must be rejected. This problem becomes worse if fewer than 2000 records are used, or if the result contains more than 3 discrete amplitudes.

Using these criteria, the result for the proximally evoked EPSP in Figure 3 can be accepted as reliable, as the average separation of adjacent amplitudes is $224 \mu \mathrm{V}$ and $\bar{v}_{1} / \sigma_{\mathrm{N}}$ is 1.94 . In the same cell, the average separation of adjacent amplitudes for the distally stimulated EPSP was $179 \mu \mathrm{V}$, giving $\bar{v}_{1} / \sigma_{N}=1.41$, and hence this result must be rejected as unreliable. A separation of $203 \mu \mathrm{V}(127 \times 1.6)$ is the minimum that could be reliably detected in this EPSP with this sample size, assuming 3 discrete amplitudes.

The analyses for 2 EPSPs evoked in another neuron are shown in Figures 8 and 9. The 10-90\% rise time of the averaged EPSP evoked hy the proximal input was $5.1 \mathrm{msec}$, and the rise time of the distal EPSP was $7.8 \mathrm{msec}$. The proximally stimulated EPSP fluctuated between 4 different amplitudes (Fig. 9C); the discrete amplitudes $(\mu \mathrm{V}) /$ probabilities were $5 / 0.17,184 / 0.36$, $381 / 0.29$, and $585 / 0.126$, and the mean separation was $193 \mu \mathrm{V}$. $\nabla_{1} / \sigma_{\mathrm{N}}$ was 2.1 , and even though 4 different amplitudes were present, our empirical testing indicated that this was very likely to be a reliable result. The same cannot be said about the result for the distally stimulated EPSP (Fig. $9 F$ ), for which the mean separation was $126 \mu \mathrm{V}$ and $\bar{\nu}_{1} / \sigma_{\mathrm{N}}$ was 1.33 . The discrete amplitudes $(\mu \mathrm{V}) /$ probabilities were $22 / 0.09,100 / 0.22,206 / 0.40$, $356 / 0.21$, and $526 / 0.06$. We suspect that a spurious peak am- plitude has been introduced at $100 \mu \mathrm{V}$, with the effect that the peaks at 206, 356, and $526 \mu \mathrm{V}$ are incorrectly located. The minimum separation that could be resolved reliably in this cell, assuming 4 discrete amplitudes, is about $180 \mu \mathrm{V}$.

Using the critcria devcloped from cmpirical testing, deconvolution results have been accepted for only 2 of the EPSPs selected for analysis (Figs. $6 C, 9 C$, and Tables 2,3). The results for the other EPSPs may be correct, but the risk in accepting them is too high. The discrete amplitudes and their probabilities given by the deconvolution procedure have been listed for each of the EPSPs in Table 3. Only discrete amplitudes with probabilities greater than 0.05 are listed, because amplitude values with probabilities smaller than this are unlikely to be correctly located. Note that the discrete amplitudes that are closest to zero $\mu \mathrm{V}$ (and therefore represent "failures") do not lie exactly at zero. This is due to small inaccuracies in the location of the discrete amplitude (particularly if it has a low probability of occurrence), and also there may be an offset common to all amplitudes if there is an extracellular synaptic field that has not been completely subtracted.

\section{Discussion}

The objectives of these experiments were to determine the mean amplitude, quantal size, and release probabilities associated with EPSPs generated at synapses formed by the collaterals of a single CA3 pyramidal neuron with a CA1 pyramidal neuron. These EPSPs were difficult to find (5/190 CA3-CA1 cell pairs tested). The reason for this low percentage of connections is not clear, although little is known about the detailed connectivity of CA3 and CA1 neurons. Extensive collateralization of Schaffer collaterals (in rat hippocampus) has been reported (Finch et al., 1983; Tamamaki et al., 1984) with one reconstructed CA3 pyramidal axon giving off 8 major collaterals in the CA1 region. These collaterals extended for more than $1.7 \mathrm{~mm}$ in the transverse plane and for more than $600 \mu \mathrm{m}$ in the longitudinal (dorsoventral) direction. Obviously the plane of the section is important in preserving transverse connectivity within the slice and our own tracing of CA3 axons in the CAl region using HRP prompted us to make the plane of section at $20^{\circ}-30^{\circ}$ to the longitudinal axis. However, the extent of the longitudinal spread of collaterals indicates that the plane of section is not the only 
A Proximal

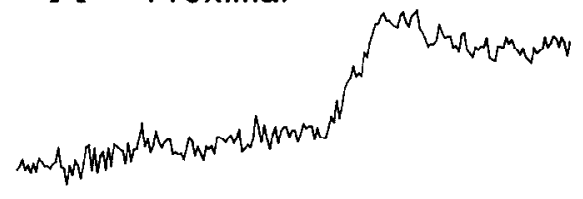

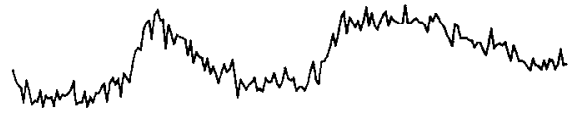

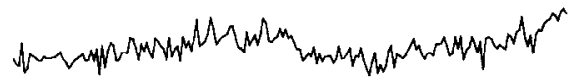

\section{B Distal}

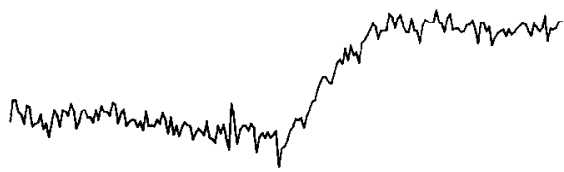

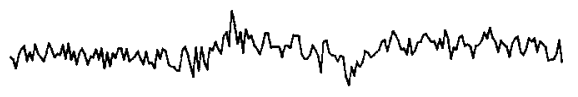

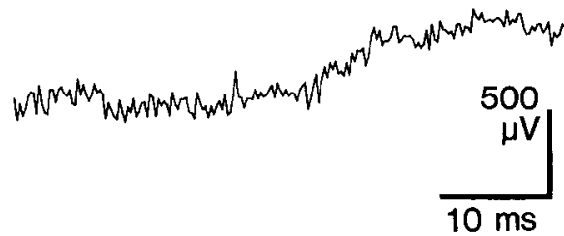

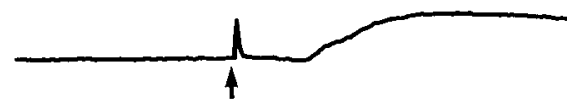

Figure 8. Examples of single records and the averages of all records for the proximally and distally stimulated EPSPs in cell 5 (Table 2). Averaged extracellular records have been subtracted. Arrows indicate the time of stimulus. factor involved, and there are many details of the extent and pattern of convergence of CA3 Schaffer collaterals onto CA1 pyramidal neurons that are yet to be determined.

The amplitudes of the 5 CA3-CA1 EPSPs ranged from 85 to $275 \mu \mathrm{V}$. Although this is a small sample, it suggests that many Schaffer collaterals must be almost simultaneously conducting impulses if this input is to bring a CAl neuron to discharge. EPSPs evoked by stimulation of stratum radiatum need to be $10-14 \mathrm{mV}$ to initiate impulses in CAl neurons in vitro (Andersen et al., 1987). Assuming linear summation of EPSPs (Langmoen and Andersen, 1983), about 100 Schaffer collaterals must be simultaneously active and converging onto the same CAl neuron to initiate an impulse. The different average peak amplitudes of these peak EPSPs could reflect differences in the number of synaptic contacts in each connection, differences in the probability of transmitter release at these contacts, and differences in the electrotonic location of synapses on the dendritic tree.

The amplitudes of 3/5 CA3-CA1 EPSPs fluctuated more than could be attributed to baseline noise. It was argued that the extent of this amplitude variation was greater than would be expected from variations in quantal EPSP amplitude alone, and there must have been variations in the number of quanta released in each trial. But the noise level was too great to determine the different amplitudes generated by the different numbers of quanta released. [This problem has also been encountered by Turner (1988).] The remaining 2 EPSPs did not vary in amplitude more than the baseline noise, but because this noise level was very high, we were unable to be certain that this was due to the absence of any intrinsic variations in EPSP amplitudes. Thus, our data cannot be used to support the assumption that the quantal EPSP has negligible variability. In contrast, quantal variability for the group la EPSP in motoneurons is very small $(\mathrm{CV}<0.05$; Jack et al., 1981). No quantal variability was included in quantal analysis of IPSPs evoked at inhibitory synapses on the Mauthner cell [Korn et al., 1982, eq. $\left(3^{\prime}\right)$ ], and
Hess et al. (1987) also assumed no quantal variance when analyzing EPSPs evoked in CA 1 neurons by stimulation of stratum radiatum. Mean quantal size and its variability are the basic building blocks in quantal analysis (Martin, 1977), and incorrect peak amplitudes and probabilities will result from any quantal analysis method if a significant quantal variability is neglected. Like others, we have assumed as a first approximation that there is no variance in the quantal EPSP for our deconvolution analysis of EPSPs evoked by stimulation of stratum radiatum.

Because of the high noise level, only 2 deconvolution results were considered to be reliable. Their interpretation is clouded by the possibility that some (or all) of the variations in peak amplitude could have resulted from intermittent excitation of collaterals. We now argue that whether or not this happens, the increments between adjacent peak amplitudes represent the quantal EPSP, where we assume by analogy with other systems (Jack et al., 1981; Korn et al., 1982; Redman and Walmsley, 1983; Walmsley et al., 1987) that the quantal EPSP results from transmitter release at one release site. Consider the result in Figure $6 C$. If we assume (1) that axons near threshold and thus intermittently stimulated are recruited independently of each other, and (2) that intermittent transmitter release at different release sites also occurs independently, then there are only 3 possible interpretations of Figure $6 \mathrm{C}$. One is that a single axon is reliably excited on all trials. It fails to release transmitter for $44 \%$ of trials, transmission occurs at only 1 release site for $44 \%$ of trials, and transmission occurs at 2 release sites for $12 \%$ of trials. The second is that a single axon is activated intermittently, and when it is excited the 3 possible outcomes are failure to release or release at either 1 or 2 release sites. The third is that 2 axons are involved, and each axon has only 1 active release site with the neuron. When both are excited, transmission occurs at the release site on each axon to give the maximum amplitude (prob. $=0.12$ ). Failures occur when one, both, or no axon is excited, but whichever occurs, no transmitter is released. The $240 \mu \mathrm{V}$ amplitude occurs when either (1) both axons are excited 
Figure 9. Deconvolution analysis for cell 5 (Table 2). Histograms are to be interpreted as for Figure 3.

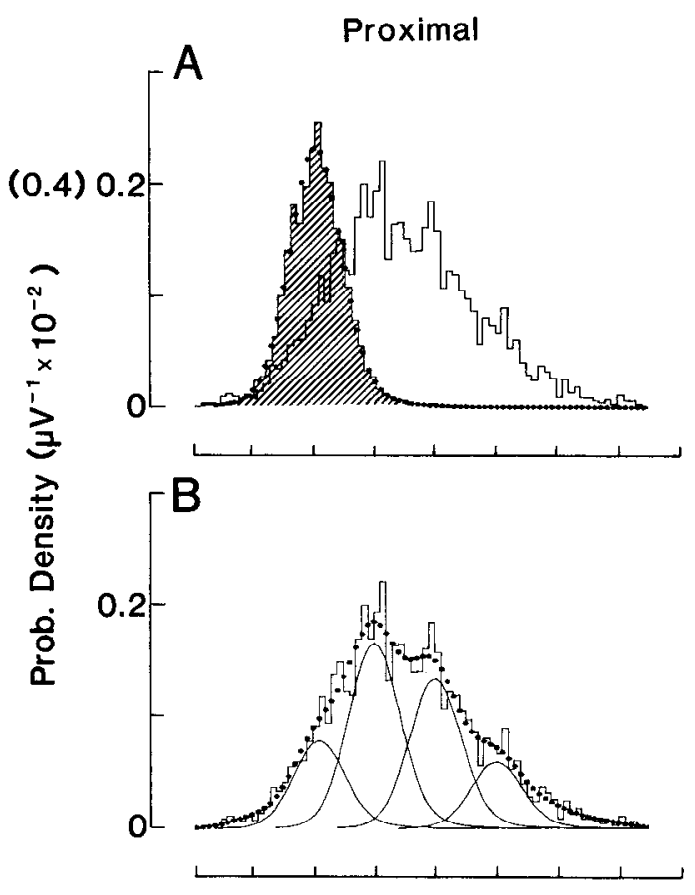

은
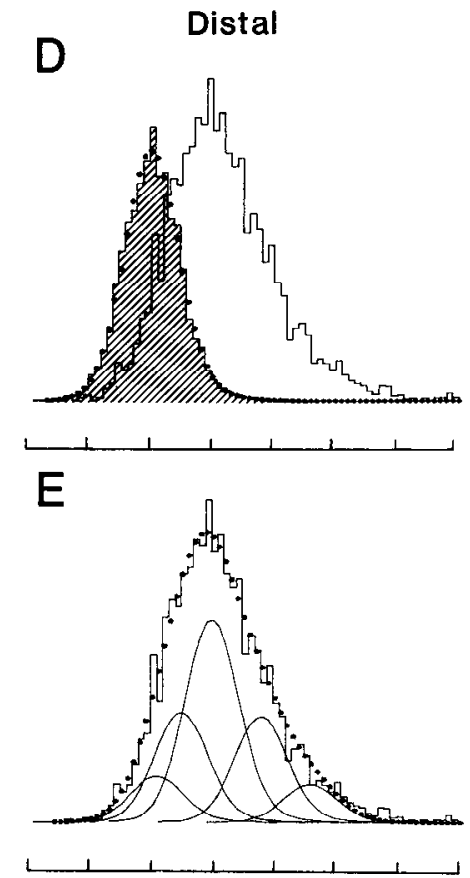

$\mathrm{F}$

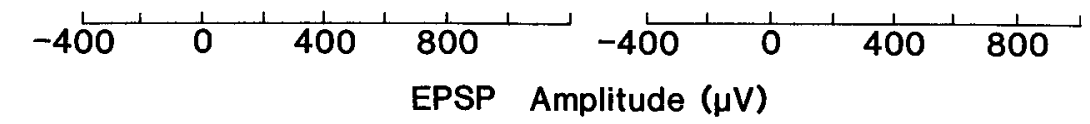

but transmitter release only occurs at 1 of the 2 release sites or (2) only 1 axon is excited and transmitter is released. A similar set of possibilities may be listed for the result in Figure $9 C$ (but involving a maximum of 3 axons). The conclusion in each case is that the increment between adjacent peak amplitudes is the quantal EPSP, which in these 2 examples was 224 and $193 \mu \mathrm{V}$. We stress that these values are likely to represent the upper limits of the quantal size, as the reason that most deconvolution results were unreliable is that the quantal amplitude usually must have been smaller than $200 \mu \mathrm{V}$.

These arguments assume that the EPSP generated at each release site will have a similar amplitude and time course at the soma. If this assumption is incorrect, then the distribution of peak amplitudes will have more peaks, and the separation be-

\begin{tabular}{|c|c|c|c|c|c|c|}
\hline $\begin{array}{l}\text { Cell } \\
\text { no. }\end{array}$ & Stimulation & $\begin{array}{l}\text { Number of } \\
\text { records }\end{array}$ & $\begin{array}{l}\bar{y} \\
(\mu \mathrm{V})\end{array}$ & $\begin{array}{l}\bar{\nu}_{1} \\
(\mu \mathrm{V})\end{array}$ & $\begin{array}{l}\sigma_{\mathrm{N}} \\
(\mu \mathrm{V})\end{array}$ & $\bar{v}_{1} / \sigma_{\mathrm{N}}$ \\
\hline \multirow[t]{2}{*}{1} & prox & 1718 & 170 & 224 & 115 & $1.94^{a}$ \\
\hline & dist & 1818 & 164 & 179 & 127 & 1.41 \\
\hline \multirow[t]{2}{*}{2} & prox & 1789 & 322 & 194 & 123 & 1.58 \\
\hline & dist & 2191 & 294 & 213 & 138 & 1.54 \\
\hline \multirow[t]{2}{*}{3} & prox & 2398 & 395 & 115 & 70 & 1.65 \\
\hline & dist & 2396 & 203 & 85 & 71 & 1.20 \\
\hline \multirow[t]{2}{*}{4} & prox & 3838 & 94 & 141 & 125 & 1.12 \\
\hline & dist & 4817 & 131 & 120 & 131 & 0.92 \\
\hline \multirow[t]{2}{*}{5} & prox & 1795 & 282 & 193 & 93 & $2.08^{a}$ \\
\hline & dist & 2298 & 227 & 126 & 95 & 1.33 \\
\hline
\end{tabular}

$\bar{v}$, mean amplitude of EPSP; $v_{1}$, mean increment between discrete amplitude values (quantal size); $\sigma_{\mathrm{N}}$, standard deviation of the noise histogram.

"EPSPs for which the deconvolution results have been accepted as reliable. 


\begin{tabular}{|c|c|c|c|c|c|c|}
\hline \multirow{2}{*}{$\begin{array}{l}\text { Cell } \\
\text { no. } \\
1\end{array}$} & \multirow{3}{*}{$\begin{array}{l}\text { Stimu- } \\
\text { lation }\end{array}$} & \multicolumn{5}{|c|}{ Discrete amplitudes $(\mu \mathrm{V})$ /probabilities } \\
\hline & & 16/0.44, & $240 / 0.44$ & $463 / 0.10$ & & $a$ \\
\hline & & $28 / 0.34$ & 196/0.54, & $386 / 0.13$ & & \\
\hline \multirow[t]{2}{*}{2} & prox & $25 / 0.08$, & $206 / 0.42$ & 395/0.34, & $608 / 0.16$ & \\
\hline & dist & 24/0.16, & $232 / 0.51$ & $480 / 0.27$, & $663 / 0.07$ & \\
\hline \multirow[t]{2}{*}{3} & prox & 179/0.11, & $273 / 0.19$ & $382 / 0.31$, & $512 / 0.23$ & $640 / 0.11$ \\
\hline & dist & $46 / 0.15$ & $142 / 0.23$ & 171/0.17, & $259 / 0.34$ & $386 / 0.07$ \\
\hline \multirow[t]{2}{*}{4} & prox & $-4 / 0.43$ & $131 / 0.42$ & $277 / 0.15$ & & \\
\hline & dist & $-17 / 0.18$ & $75 / 0.35$ & 196/0.37, & $343 / 0.10$ & \\
\hline \multirow[t]{2}{*}{5} & prox & $5 / 0.17$ & $184 / 0.36$ & $381 / 0.29$ & $585 / 0.13$ & $a$ \\
\hline & dist & $22 / 0.09$, & $100 / 0.22$ & $206 / 0.40$ & $356 / 0.21$ & $526 / 0.06$ \\
\hline
\end{tabular}

"EPSPs for which the deconvolution results have been accepted as reliable.

tween some of them will be unresolvable by deconvolution. In practice, this problem may not be as bad as it seems. For example, if the correct solution in Figure $7 C$ involved 3 different amplitudes near $200 \mu \mathrm{V}$ (corresponding to 3 different quantal sizes) and similarly 3 different amplitudes around $400 \mu \mathrm{V}$, the deconvolution result would be almost the same as in Figure $7 C$. However, if the difference in quantal sizes were sufficiently large to cause a more uniform dispersal of peak amplitudes between 0 and $600 \mu \mathrm{V}$, the deconvolution result would be meaningless.

The quantal EPSP calculated by Hess et al. (1987) is larger than that determined here $(240 \pm 140$ to $297 \pm 129 \mu \mathrm{V}$, mean $\pm \mathrm{SD}$, the size depending on the method of calculation). The various formulas used by Hess et al. (1987) are all model dependent (binomial or Poisson), and they have been applied to histograms of EPSP amplitudes (constructed from about 100 records) containing distinct peaks and troughs with approximately regular spacing. We never observed histograms where this occurred, even though the noise levels in our experiments were similar to those reported by Hess et al. (1987). These differences remain puzzling, but if the quantal size was consistently greater than about $200 \mu \mathrm{V}$, as reported by Hess et al. (1987), our deconvolution methods would have routinely extracted it. In fact, our lowest noise recordings, with $\sigma_{\mathrm{N}}$ about 70 $\mu \mathrm{V}$ (Table 2, cell 3) would have resolved discrete amplitudes separated by $110 \mu \mathrm{V}$ or more.

In this kind of analysis, the question usually surfaces about which type of probability distribution is appropriate to describe the probabilities of the different peak amplitudes, with its attendant implications for the probabilities of release at different release sites. The results in Figures $6 C$ and $9 C$ could be fitted by a simple binomial ( $n=3$ for Fig. $6 C$ and $n=6$ for Fig. $9 C$ ) to an acceptable confidence limit using the $\chi^{2}$ test, but a compound binomial gave a slightly better fit (using this test) to the results of Figure $6 C$. This means that if the 2 deconvolved results are not contaminated by intermittent excitation of axons, the probabilities of release are likely to be similar at the release sites involved. No such conclusion can be drawn if axons are intermittently excited.

In spinal motoneurons, EPSPs evoked by impulses in group la axons or in descending monosynaptic connections have quantal amplitudes (at the soma) that are independent of synaptic location (Jack et al., 1981; Edwards et al., 1983; Harrison et al., 1985). We were interested to find out if this also occurred for synaptic inputs to CA1 neurons. The 2 EPSPs that were reliably deconvolved were proximal inputs, and we were unable to calculate the quantal size for the distal inputs to these same neurons. But we would have been able to do so if the quantal size of the distal EPSPs had been the same size (or larger) as for the proximal EPSPs. For these 2 neurons we conclude that the quantal EPSP is smaller for distal synapses than it is for proximal synapses.

Finally, we note that there are important anatomical details of the Schaffer collateral-CAl pyramidal neuron connection that have not been described. Although it is known that at least $95 \%$ of the synapses formed on spines of apical dendrites have only one postsynaptic density (Lee et al., 1980), there has been no report on the number of contacts made by a single Schaffer collateral with individual CAl pyramidal neurons, nor on the frequency and spatial location of connections made by one collateral with pyramidal neurons. When this information is available, and when the noise level of intracellular recordings from CAl neurons is improved, it should be possible to perform the same detailed analysis of transmitter release processes at synapses on Schaffer collaterals as has been achieved for inhibitory synapses on Mauthner cells and for group la synapses on motoneurons, and for synapses made by primary muscle afferents on dorsal spinocerebellar tract neurons.

\section{References}

Andersen, P. (1975) Organization of hippocampal neurons and their interconnections. In The Hippocampus, Vol. 1, R. L. Isaacson and K. H. Pribram, eds., pp. 155-175, Plenum, New York.

Andersen, P., H. Silfvenius, S. H. Sundberg, and O. Sveen (1980) A comparison of distal and proximal dendritic synapses on CAl pyramids in guinea-pig hippocampal slices in vitro. J. Physiol. (Lond.) 307: 273-299.

Andersen, P., J. Storm, and H. V. Wheal (1987) Thresholds of action potentials evoked by synapses on the dendrites of pyramidal cells in the rat hippocampus in vitro. J. Physiol. (Lond.) 383: 509-526.

Clements, J. D., I. D. Forsythe, and S. J. Redman (1987) Presynaptic inhibition of synaptic potentials evoked in cat spinal motoneurones by impulses in single group Ia axons. J. Physiol (Lond.) 383: 153169.

Dempster, A. P., N. M. Laird, and D. B. Rubin (1977) Maximum likelihood from incomplete data via the EM algorithm. J. R. Statist. Soc. B 39: 1-21.

Edwards, F. R., J. J. B. Jack, and D. M. Kullmann (1983) The relationship between amplitude and time course of single fibre group Ia 
excitatory post-synaptic potentials in cat spinal motoneurones. $\mathrm{J}$. Physiol. (Lond.) 345: 58P.

Finch, D. M., N. L. Nowlin, and T. L. Babb (1983) Demonstration of axonal projections of neurons in the rat hippocampus and subiculum by intracellular injection of HRP. Brain Res. 271: 201-216.

Hasselblad, V. (1966) Estimation of parameters for a mixture of normal distributions. Technometrics $8: 431-444$.

Harrison, P. J., J. J. B. Jack, and D. M. Kullmann (1985) The components of monosynaptic excitatory post-synaptic potentials evoked in cat lumbosacral motoneurones by impulses in descending fibres. J. Physiol. (Lond.) 365: 32P.

Hess, G., U. Kuhnt, and L. L. Voronin (1987) Quantal analysis of paired pulse facilitation in guinea pig hippocampal slices. Neurosci. Lett. 77: 187-192.

Jack, J. J. B., S. J. Redman, and K. Wong (1981) The components of synaptic potentials evoked in cat spinal motoneurones by impulses in single group Ia afferents. J. Physiol. (Lond.) 321: 65-96.

Johnson, N. L., and S. Kotz (1970) Continuous Univariate Distributions, 2nd ed., Houghton-Mifflin, Boston.

Korn, H., A. Mallet, A. Triller, and D. S. Faber (1982) Transmission at a central inhibitory synapse. II. Quantal description of release, with a physical correlate for binomial n. J. Neurophysiol. 48: 679-707.

Langmoen, I. A., and P. Andersen (1983) Summation of excitatory postsynaptic potentials in hippocampal pyramidal cells. J. Neurophysiol. 50: 1320-1329.

I ee, K. S., F. Schottler, M. Oliver, and G. Lynch (1980) Brief bursts of high frequency stimulation produce two types of structural change in rat hippocampus. J. Neurophysiol. 44: 247-258.

Ling, L., and D. J. Tolhurst (1983) Recovering the parameters of finite mixtures of normal distributions from a noisy record: An empirical comparison of different estimating procedures. J. Neurosci. Methods 8: 309-333.

Martin, A. R. (1966) Quantal nature of synaptic transmission. Physiol. Rev. 46: 51-66.

Martin, A. R. (1977) Junctional transmission. II. Presynaptic mechanisms. In Handbook of Physiology, Vol. 1, Sec. 1, E. Kandel, ed., pp. 329-355, American Physiological Society, Bethesda, MD.

McLachlan, E. (1978) The statistics of transmitter release at chemical synapses. In International Review of Physiology. Neurophysiology III, Vol. 17, R. Porter, ed., pp. 49-117, University Park Press, Baltimore, MD.

Redman, S., and B. Walmsley (1983) Amplitude fluctuations in synaptic potentials evoked in cat spinal motoneurones at identified group Ia synapses. J. Physiol. (Lond.) 343: 135-145.

Tamamaki, N., K. Watanabe, and Y. Nojko (1984) A whole image of the hippocampal pyramidal neuron revealed by intracellular pressure injection of horseradish peroxidase. Brain Res. 307: 336-340.

Turner, D. (1988) Waveform and amplitude characteristics of evoked responses to dendritic stimulation of CAl guinea-pig pyramidal cells. J. Physiol. (Lond.) 395: 419-439.

Walmsley, B., F. R. Edwards, and D. J. Tracey (1987) The probabilistic nature of synaptic transmission at a mammalian excitatory synapse. J. Neurosci. 7: 1037-1046.

Westrum, L. E., and T. W. Blackstad (1962) An electron microscopic study of the stratum radiatum of the rat hippocampus (regio superior, CA1) with particular emphasis on synaptology. J. Comp. Neurol. 119. 281-309.

Wong, K., and S. Redman (1980) The recovery of a random variable from a noisy record with application to the study of fluctuations in synaptic potentials. J. Neurosci. Methods 2: 389-409. 